\title{
Etica e progettazione europea. Uno studio di caso
}

Ethics and European research funding. A case study

\section{Roberta Raffaetà}

\section{(2) OpenEdition}

\section{Journals}

\section{Edizione digitale}

URL: https://journals.openedition.org/aam/3994

DOI: 10.4000/aam.3994

ISSN: 2038-3215

\section{Editore}

Dipartimento Culture e Società - Università di Palermo

\section{Notizia bibliografica digitale}

Roberta Raffaetà, «Etica e progettazione europea. Uno studio di caso», Archivio antropologico mediterraneo [Online], Anno XXIV, n. 23 (1) | 2021, online dal 30 juin 2021, consultato il 02 juillet 2021. URL: http://journals.openedition.org/aam/3994 ; DOI: https://doi.org/10.4000/aam.3994

Questo documento è stato generato automaticamente il 2 juillet 2021

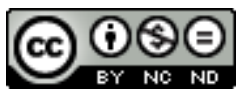

Archivio antropologico mediterraneo è distribuita con Licenza Creative Commons Attribuzione - Non commerciale - Non opere derivate 4.0 Internazionale. 


\title{
Etica e progettazione europea. Uno studio di caso
}

\author{
Ethics and European research funding. A case study
}

\author{
Roberta Raffaetà
}

\section{Introduzione}

1 Gli aspetti etici della ricerca, fino a qualche tempo fa, erano per me principalmente una questione di responsabilità individuale nei confronti delle persone che partecipavano alle mie ricerche, e solo secondariamente come qualcosa con un'incidenza sulla disciplina. Dopo aver ottenuto un finanziamento europeo i cui meccanismi sono altamente formalizzati, mi sono resa conto che pensare all'etica era anche pensare a dove sta andando la nostra disciplina, l'accademia e i rapporti tra le persone.

In questo articolo opero una ricostruzione etnografica del processo relativo alla formulazione degli aspetti etici di un progetto ERC Starting grant che coordinerò a partire da settembre 2021. La descrizione etnografica permette di addentrarsi in alcuni dettagli che consentono di decostruire l'etica come un campo che pertiene strettamente alla dimensione legale o amministrativa, mostrando come tali dimensioni siano intrecciate a elementi socio-politici locali, nazionali, europei e globali, che trovano risonanza nelle biografie dei/lle ricercatori/ici. Questi vanno dalla precarietà accademica ormai endemica (Stoica et al. 2019) che spinge verso finanziamenti individuali, alle macro-politiche di un'università sempre più di stampo neoliberista (Slaughter Rhoades 2004), organizzata secondo logiche e protocolli aziendali (Etzkowitz 2013). La comprensione di tali aspetti, e delle loro relazioni, è essenziale al fine di sviluppare una consapevolezza circa la complessità dei discorsi sull'etica, legarli a una riflessione sullo statuto della disciplina ed avviare un attivismo collettivo su questi temi nel tentativo di trovare delle soluzioni che rendano conto delle specificità della disciplina antropologica.

3 Prima di procedere, bisogna innanzitutto operare una riflessione terminologica. L'etica, secondo il dizionario Treccani, è «ogni dottrina o riflessione speculativa intorno al 
comportamento pratico dell'uomo, soprattutto in quanto intenda indicare quale sia il vero bene e quali i mezzi atti a conseguirlo, quali siano i doveri morali verso sé stessi e verso gli altri, e quali i criteri per giudicare sulla moralità delle azioni umane». L'etica, in senso generale, è quindi un insieme di riflessioni e considerazioni ampie sul comportamento. In ambito di progettazione europea, invece, quando si parla di 'etica' ci si riferisce a un processo tecnico/amministrativo volto alla legalizzazione della propria ricerca. Per esempio, alcune delle domande comuni tra coordinatori/ici di progetto o tra questi/e e il personale ammnistrativo sono «Hai già fatto l'etica?», «Quando arriva l'etica?» ecc... All'interno di questo spazio discorsivo, il GDPR occupa una posizione di massimo rilievo, dato che viene considerato come il principale riferimento normativo. Questa è una riduzione metonimica che ha delle conseguenze, come ben sottolineato da Elena Bougleux nelle sedute di preparazione all'incontro SIAC (si veda Bougleux in questo numero). Considerare i dati come l'unità di riferimento nei discorsi sull'etica rischia di oscurare altre riflessioni ma, soprattutto, rischia di reificare lo stesso concetto di 'dati' non solo come qualcosa di oggettivo e tangibile 'là fuori' ma anche di costruire la loro esistenza nei termini definiti dal GDPR, termini che verranno analizzati in alcuni aspetti nelle prossime sezioni.

4 Questo articolo è quindi un tentativo di ricollegare il GDPR con l'etica, illustrando alcuni, tra i tanti, nodi problematici - ma anche generativi - del processo di validazione etica. Nel fare ciò, cerco di dare rilevanza a due aspetti principali. Uno è il significato da dare all'etica, come insieme di norme rigide oppure come un incoraggiamento ad aprire un discorso sull'etica, e quale è il nostro posizionamento in tale dibattito in quanto antropologi/ghe e in quanto associazioni. L'altro aspetto è quello delle peculiarità della ricerca antropologica rispetto alla definizione normativa di 'dati'.

Per ancorare etnograficamente le riflessioni che svilupperò in quest'articolo, è necessario un cenno alle specificità della tipologia progettuale a cui mi riferisco e descrivere per sommi capi le caratteristiche principali del progetto ERC in oggetto. Ciò permetterà al lettore di valutare criticamente la specifica angolatura con cui io mi avvicino alle questioni etiche, e quindi apprezzare limitazioni e bias di quanto esporrò.

6 Le opportunità di finanziamento per la ricerca e l'innovazione della Commissione Europea prevedono due macro gruppi principali: i Funding programmes and open calls di cui fa parte Horizon 2020, the Cohesion Fund, LIFE, ERDF, ESIF and RFCS ecc... e il gruppo delle Fellowship and individual research grants, composto da borse Marie Curie e ERC. ERC sta per European Research Council (Consiglio Europeo della Ricerca), la cui missione è quella di «incoraggiare la ricerca della massima qualità in Europa attraverso finanziamenti competitivi e sostenere la ricerca di frontiera promossa dai ricercatori in tutti i campi della ricerca, sulla base dell'eccellenza scientifica»' ${ }^{1}$. L'ERC finanzia progetti presentati da ricercatori individuali (e non da atenei o consorzi di atenei) senza che questi debbano aderire o rispondere a tematiche di ricerca identificate a priori. I criteri di valutazione sono primariamente la qualità scientifica e il contributo per l'avanzamento della disciplina. L'impatto sociale della ricerca proposta è sicuramente considerato, ma in misura minore rispetto ad altre linee di finanziamento. Le borse erogate (da un minimo di $1500000 \mathrm{eu}$ a un max di $2500000 \mathrm{eu}$ ) servono a coprire i costi per la creazione di un gruppo di ricerca e i costi associati alla ricerca (strumentazione, viaggi, conferenze, ecc...). In tale cornice, gli aspetti etici rivestono un ruolo importante. Basti pensare che, dopo la comunicazione dell'assegnazione del 
finanziamento, la sua conferma avviene solo dopo vari mesi dato che è vincolata all'esito positivo di un denso procedimento di valutazione etica.

7 Gli aspetti etici del progetto da me presentato sono legati alla natura della ricerca proposta. Il progetto ${ }^{2}$ propone un'etnografia che parte da laboratori di ricerca in metagenomica ${ }^{3}$ situati in varie parti del mondo (Stati Uniti e tre luoghi del Sud globale) per poi continuare nel contesto socio-culturale più ampio in cui si trovano i laboratori, attraverso contatti con comunità rilevanti che stanno all'incrocio tra salute pubblica, industria e protezione dell'ambiente. Il gruppo di ricerca che costituirò, ed io, ci relazioneremo, soprattutto con scienziati, medici, policy-makers, attivisti ecc... L'osservazione partecipante avverrà sia all'interno dei laboratori che in contesti più ampi. Relazionarsi principalmente con scienziati, da una parte, alleggerisce gli aspetti etici della ricerca perché gli scienziati sono persone maggiorenni (escludendo così tutta una serie di criticità etiche legate alla ricerca con minori), né vengono considerati come una comunità particolarmente vulnerabile. Dall'altra, però, fare ricerca nei laboratori significa che l'accesso a questi sarà rigidamente formalizzato e che bisognerà prestare attenzione alla formulazione di accordi preliminari (per es., il 'non disclosure agreement') che regolano la protezione della proprietà intellettuale e la diffusione di certi dati. Nella sezione che segue, ricostruirò le dinamiche e i vari passaggi per l'approvazione etica che mi hanno vista impegnata per vario tempo, soffermandomi su un aspetto particolare, che è quello del trattamento di 'categorie speciali di dati'.

\section{Panico da 'ethics'}

8 In ambito di progettazione europea, nel caso specifico degli ERC, per completare la domanda di finanziamento e poterla inviare è necessario compilare un modulo standard per tutte le discipline con una serie di domande a risposta chiusa (Sİ/NO) il cui obiettivo è identificare il grado di vulnerabilità, da un punto di vista etico, del progetto. Nel caso si risponda affermativamente a certe domande (per esempio, nel caso di ricerche antropologiche quasi sempre si risponde «Sì» alla domanda: «La ricerca include partecipanti umani?»), bisogna allegare un documento aggiuntivo che descriva in forma narrativa le misure che si pensa di adottare per gestire gli aspetti etici del progetto. Tutta l'operazione, nel mio caso, prese un paio di ore al massimo. I problemi vennero dopo.

Nel caso di assegnazione del finanziamento, dopo qualche mese dalla comunicazione (nel mio caso dopo due mesi), il/la futuro/a PI (Principal Investigator, il/la coordinatore/ice del progetto) riceve un documento chiamato Ethics Screening Report che dettaglia tutte le possibili problematiche etiche relative al progetto in oggetto e le relative richieste. Queste possono richiedere un chiarimento narrativo oppure di produrre documentazione di supporto. Il report viene elaborato da esperti dell'unità Ethics Review and Expert Management dell' European Research Council Executive Agency (ERCEA). Nel mio caso, il report contava 8 pagine e includeva tre categorie principali: «Humans», «Protection of personal data» e «Non EU countries». In questo ariticolo mi soffermerò solo su alcune voci delle prime due categorie. Il/la PI deve rispondere, nell'arco di tempo di tre settimane a tali richieste, producendo un documento chiamato Reply to the Ethics Report. Questo è un passo fondamentale e che deve venir fatto con la massima cura perché, come già accennato, la conferma dell'effettiva assegnazione del finanziamento da parte dell'Unione Europea attraverso la firma del grant agreement 
(contratto che regola i rapporti tra il beneficiario e l'ERCEA) può avvenire solo dopo l'accettazione della risposta del/la PI.

10 Nonostante vari colleghi mi avessero avvisato in anticipo circa il grande impegno richiesto per ottenere l'approvazione della parte etica, io non mi persi d'animo e presi il compito come un'occasione per approfondire alcuni aspetti etici di cui, negli anni, ero riuscita solo a sfiorare la superficie. Per esempio, dal giorno in cui un'università mi aveva assegnato un incarico di ricerca come assegnista previa firma di un documento in cui si dichiarava che i dati che producevo sarebbero stati proprietà dell'università e che dovevo distruggerli dopo il mio incarico, avevo cercato se esistesse un qualche documento che regolava, a livello europeo, la proprietà e il trattamento dei dati in antropologia. Trovai l'EASA's Statement on Data Governance in Ethnographic Projects, sviluppato dall'EASA Ethics Network, presieduto da Peter Pels (Leiden University). Il documento è un estratto di quello che viene chiamato Leiden Statement on Data Management and Anthropology (Pels et al. 2018). Lo Statement in questione consta di una pagina, che stampai e attaccai al muro del mio ufficio, come un feticcio protettivo nei confronti di un'accademia che percepivo come cannibale.

11 Per sviluppare una risposta al report dell'ERCEA partii proprio dal Leiden Statement. A ciò aggiunsi due articoli comparsi nel 2019 nella rivista Ethnography (de Koning et al. 2019; Dilger et al. 2019). Solo in un secondo momento - quando ormai avevo già inviato la mia risposta all'ERCEA - venni a conoscenza di un consistente documento (75 pagine) promosso dalla Comunità Europea dal titolo Research Ethics in Ethnography/Anthropology. L'autore di tale scritto è Ron Iphofen, un consulente indipendente specializzato negli aspetti etici della ricerca con una formazione nelle scienze sociali, soprattutto nella sociologia della medicina ${ }^{4}$. Nonostante un approccio a tratti un po' troppo positivista (con allusioni a una supposta 'oggettività' della situazione da studiare che il/la ricercatore/ice potrebbe 'disturbare'), il documento è sicuramente prezioso e offre interessanti spunti di riflessione tesi a mostrare come, nel campo dell'etica e soprattutto in riferimento a una disciplina come l'antropologia, non si possa avere un atteggiamento manicheo che distingue in maniera netta tra giusto e sbagliato. Mi immersi nell'analisi di questi testi e riemersi con un documento di risposta alla valutazione ERCEA di circa 13 pagine. Il lavoro prese circa due settimane a pieno ritmo. La mia risposta non solo rispondeva alle richieste degli esperti ERCEA, ma - in piena sintonia con il tono del Leiden Statement - avanzava degli argomenti epistemologici e politici che mettevano in luce le particolarità della pratica antropologica ed etnografica.

12 Alcuni di questi aspetti verranno discussi nella prossima sezione, mentre in questa mi soffermerò sulla mia risposta alla richiesta dei valutatori di chiarire se intendessi raccogliere e 'processare' "special categories of data" (categorie speciali di dati). Questa è una particolare tipologia di dati che, secondo l'art.9 del GDPR, possono rivelare «ethnic origin, political opinions, religious or philosophical beliefs, trade union membership, data concerning health and data concerning a sex life or sexual orientation». Nel caso si affermi di raccogliere questo tipo di dati, viene richiesto di giustificare tale scelta. La mia risposta metteva in luce che la raccolta di tali dati sta spesso alla base di una ricerca antropologica e che la loro raccolta è permessa perché come sottolineato più volte nel Leiden Statement - rientra nella deroga speciale che tutela la libertà accademica di espressione, inclusa nell'art. 85(2) del GDPR. 
13 Ero molto fiera di ciò che avevo prodotto e lo spedii, per averne un riscontro, alle due responsabili dell'ufficio di supporto alla ricerca dell'università con cui avevo presentato la domanda. Il mio entusiasmo venne da queste prontamente troncato. Esse mi spiegarono che alcune delle riflessioni che avanzavo non erano pertinenti e, anzi, rischiavano di insospettire e confondere i valutatori, rendendo il processo più lungo $\mathrm{e}$ laborioso. Il loro consiglio era di adeguarmi e adottare il lessico dei valutatori senza perdermi troppo nelle sottigliezze concettuali ed epistemologiche. Mi suggerirono di andare dritta al cuore delle richieste dei valutatori fornendo loro le rassicurazioni che cercavano in maniera netta e non opinabile. Inoltre, mi dissero che l'articolo del GDPR a cui mi appellavo per garantire la libertà di espressione accademica (art. 85(2)) non era recepito dal codice deontologico che regola la ricerca scientifica in Italia e che quindi dovevo cancellare le parti in cui lo citavo. Infatti, per quanto il GDPR sia un regolamento europeo, e io fossi finanziata dalla $\mathrm{CE}$, l'università che mi ospitava era in Italia e quindi era necessario fare riferimento alla legislazione italiana.

La soluzione che loro mi offrivano per poter condurre una ricerca etnografica e, al tempo stesso, rispondere alle richieste del GDPR era quella di dichiarare che avrei presentato ai/lle partecipanti alla ricerca ben cinque documenti. Oltre ai tre 'tradizionali' (documento informativo, consenso informato e dichiarazione di cessione dei diritti in caso di raccolta di immagini/video/audio), ne avrei dovuti aggiungere altri due: un documento che informava circa le modalità di 'processamento' delle categorie speciali di dati personali, enfatizzando che tale operazione avrebbe costituito maggiori rischi per i/le partecipanti, e il consenso informato legato a tale documento.

Come ogni antropologo/a può ben immaginare, l'idea di presentarmi con cinque documenti mi terrorizzava. E ciò non perché io non voglia essere trasparente con le persone che parteciperanno alla ricerca o sottovaluti l'importanza degli aspetti etici. Al contrario. Presentare cinque documenti può bloccare la relazione etnografica, spaventando gli/le interlocutori/ici ma anche infastidendoli/le. Cinque documenti rappresentano un eccesso di informazione, è una misura sproporzionata rispetto al rischio previsto che non mette al primo posto l'interesse del partecipante alla ricerca. Inoltre, come più volte sottolineato anche nel Leiden Statement e nell'altra letteratura citata prima, è limitante affidare a una firma un aspetto così delicato come l'etica della ricerca antropologica. Questa, per via della stessa natura del metodo etnografico, deve essere negoziata e verificata durante tutto l'arco temporale della ricerca e, a livello relazionale, va ben oltre la firma di un documento. La firma di un consenso informato, infatti, non è vincolante legalmente perché è necessario assicurare ai/lle partecipanti alla ricerca la possibilità di ritirarsi da questa in qualsiasi momento e senza dover fornire alcuna giustificazione. La firma e la presentazione di tutti quei documenti rischiano quindi di costituire un rituale a vantaggio e rassicurazione di un apparato amministrativo che, nella sua performatività, legittima una logica che poco ha a che fare con una sensibilità, ma anche un'epistemologia, antropologiche.

A queste riflessioni si accompagnava però una crescente ansia. Il tempo passava, pochi giorni erano ancora rimasti per presentare la risposta all'ERCEA, vincolante per l'ottenimento del finanziamento. La notte facevo strani incubi, tipo quello di essere l'unica persona al mondo a non aver ottenuto il finanziamento per non aver passato la fase etica, o averla spedita in ritardo. La vincita di un ERC è sicuramente un grande privilegio che - soprattutto per la fascia più bassa, quella Starting - libera da altri incubi, quello della precarietà accademica senza un orizzonte definito che affligge, 
ormai in maniera cronica, molti/e ricercatori/ici. La vincita di un ERC è un modo - per alcuni praticamente l'unico - per raggiungere una stabilizzazione nel sistema accademico. Ma è proprio questo salto repentino dalla marginalità più profonda alle luci della ribalta - e associate responsabilità - a provocare una pressione che inizialmente può essere difficile da gestire. Vista la scala del finanziamento ottenuto $\mathrm{e}$ gli interessi correlati (istituzioni, atenei, ricercatori, ecc.), avevo la sensazione di essere entrata in un meccanismo più grande di me. Ero quindi divisa tra il proseguire la 'battaglia' per vedere riconosciute le peculiarità della pratica antropologica e il rinunciare a ciò in nome della conclusione snella dei procedimenti preliminari al progetto. Oltre alla letteratura già citata, non avevo familiarità con nessun gruppo di sostegno a cui appellarmi in materia etica e mi sentivo abbastanza sola. Le mie uniche alleate - le due rappresenti dell'ufficio di supporto alla ricerca - mi consigliavano di lasciar perdere la battaglia e adeguarmi. Mi facevano anche capire che, se non avessi modificato il mio report nella direzione consigliata, il progetto avrebbe rischiato di non essere approvato dalla commissione etica dell'ateneo e dal DPO (Data Protection officer, la persona che, all'interno di ogni ateneo controlla e supervisiona il trattamento di dati personali). Se si è nuovi dell'argomento - come lo ero io - è difficile portare avanti da soli e in poco tempo un argomento tanto politicamente carico e complesso. Scelsi quindi una via di mezzo tra le due opzioni: lasciai (contro il parere delle due amministrative) le riflessioni epistemologiche e l'accenno al Leiden Statement e all'art. 85(2). In merito a quest'articolo, sottolineai che «This derogation has been designed to guarantee the critical social value of humanities and social sciences research». Dall'altra, però, acconsentii ad includere un paragrafo in cui dichiaravo che avrei presentato i cinque documenti per la protezione delle categorie di dati speciali. Alla ricerca di una qualche scappatoia, mi misi a studiare in maniera approfondita il GDPR e aggiunsi alla dichiarazione precedente: «Revelation of sensitive data will also comply with the exceptions to article 9(1) listed in the article 9(2), especially with reference to point $a, e, g, j »$. Questi commi dell'art. 9(2) sono delle eccezioni alla proibizione relativa al processamento delle categorie speciali di dati, in particolare se in presenza di consenso (a), se resi pubblici dalla persona a cui appartengono (e), e in nome dell'interesse pubblico di tali dati $(g, j)$. Circa un mese dopo, ricevetti una email dalla persona dell'ERCEA che valutava la mia pratica. L'email mi comunicava che la mia risposta era stata accettata, previo invio, entro i tempi dichiarati, di alcuni documenti non ancora allegati. Tirai un respiro di sollievo, anche se sapevo che la 'battaglia' non era ancora finita.

\section{GDPR in contesto}

17 È però importante comprendere le dinamiche socio-politiche locali, nazionali e europee che hanno determinato il tipo di supporto che ricevetti nell'ateneo con cui presentai la proposta ERC. Bisogna tenere conto che la pubblicazione del GDPR ha portato molte università ad avere un comportamento precauzionale nei confronti degli aspetti etici della ricerca dato che queste compaiono come l'entità legale di ogni progetto finanziato. Esse non devono solo garantire il rispetto delle regole, ma diventano anche l'ente verso cui ERCEA/CE possono appellarsi in caso di infrazione. Più o meno ogni università, almeno a partire dall'entrata in vigore del GDPR, ha una commissione etica e un DPO. Il personale amministrativo ha un doppio ruolo: da una parte supporta il/la PI 
ma dall'altra cerca di tutelare gli interessi dell'ateneo. Questo doppio ruolo può avere varie configurazioni.

Nel mio caso, proprio nelle settimane in cui dovevo redigere il report sull'etica stavo anche valutando la possibilità di trasferire il progetto presso un'altra università. Questa è un'eventualità abbastanza frequente nel caso degli ERC: questo finanziamento è legato al/la ricercatore/ice e non all'università e chi vince un ERC riceve normalmente proposte da parte di altri atenei. Il dipartimento con cui presentai il progetto non era particolarmente interessato a ospitare un progetto antropologico perché ha una forte tradizione sociologica; inoltre il dipartimento non era neppure aperto a considerare la mia richiesta di potenziamento dell'attuale offerta antropologica sia nella ricerca che nella didattica. Insomma, agli occhi della macchina amministrativa di quell'ateneo il mio progetto non era particolarmente importante e, comprensibilmente, era percepito come un peso non solo aggiuntivo al già ingente lavoro ordinario, ma anche poco redditizio perché esisteva la concreta possibilità che di tale lavoro, infine, avrebbe beneficiato un altro ateneo. Le due persone che mi supportavano erano comunque molto gentili con me, ma avevano un carico di lavoro superiore alle capacità di gestione: negli ultimi tempi, l'ufficio di supporto alla ricerca - per motivi congiunturali (maternità e trasferimenti) e strutturali - si trovava sottodimensionato. Esse erano sicuramente anche molto competenti rispetto alle procedure ammnistrative ma non così avvezze alle specificità della ricerca antropologica, da queste accomunata alla sociologia.

Dopo profonde riflessioni e varie negoziazioni, optai per Ca' Foscari, un'università con un gruppo antropologico stimolante e intenzionato a portare avanti un progetto per lo sviluppo della disciplina. Inoltre, Ca' Foscari ha un eccezionale organizzazione per quanto riguarda tutto il comparto di supporto alla ricerca, a cui l'ateneo dà molta importanza e a cui vanno molti fondi (anche quelli derivanti da ERC). Finita in un meccanismo più grande di me - l'ERC - sentii l'esigenza - per potere dedicarmi alla ricerca con la giusta serenità - di farmi supportare da un ateneo capace di gestire e interpretare ad hoc le parti amministrative del progetto. Queste premesse hanno una ricaduta importante anche sul tipo di relazione che ho instaurato con le persone e gli enti che, all'interno dell'ateneo, mi aiutano a gestire il mio progetto dal punto di viste degli aspetti etici. A Ca' Foscari, per il momento, non ho trovato solo un personale amministrativo altamente qualificato ma anche curioso e aperto a comprendere e supportare le complessità disciplinari e specifiche di un progetto.

A Ca' Foscari esiste una commissione etica, un DPO e un Data Monitoring Board che si occupa specificatamente dei temi inerenti il trattamento e la protezione dei dati personali. Presi appuntamento con $\mathrm{Marco}^{5}$, esperto giuridico in materia di protezione dei dati personali, per discutere assieme a lui i miei dubbi su alcuni passaggi della mia risposta all'ERCEA e anche per valutare la possibilità di rinegoziarli. Egli convenne con me che presentare cinque documenti era troppo e lesivo della libertà altrui. Ca' Foscari ha un documento unico in cui viene incluso anche il trattamento di categorie speciali di dati, con questa frase: «With regards to the processing of special categories of personal data, the legal basis is represented by art. 9.2.a) of the Regulation ("explicit consent of the data subject") in connexion with art. 7.2 of "Regole deontologiche"».

Marco confermò comunque che il codice deontologico che regola i trattamenti di dati personali per scopi statistici e scientifici in Italia non ha recepito l'art. 85(2). Pose tuttavia alla mia attenzione anche il fatto che tale articolo si riferisce più che altro alla 
libertà accademica di espressione legata all'insegnamento o alla deliberazione di idee personali. Per quanto invece pertiene la libertà di espressione nella ricerca, mi disse, l'articolo più adeguato a tutelare la libertà è l'art.89. La dott.ssa Barrera, durante l'incontro SIAC, aveva già accennato a tale articolo. Il problema è che, secondo le analisi che avevo condotto sul codice deontologico nazionale che regola il trattamento dei dati a fini statistici o di ricerca scientifica, questo riceve l'art. 89(1) che è quello che esplicita i diritti delle persone alla protezione dei dati, ma non gli art. $89(2,3)$ che sono quelli, appunto, che prevedono alcune eccezioni, tra cui quelle per fini di ricerca. Marco, comunque, mi rassicurò, delineandomi uno scenario in divenire per quanto riguarda $\mathrm{i}$ regolamenti sul trattamento dei dati e anche su quelli deontologici in Italia. Mi disse che nel 2021 è prevista la pubblicazione delle linee guida europee relative all'European Data Protection Board (EDPB) e che il Garante della Privacy italiano sta mettendo in programma varie revisioni dei codici deontologici. In questo contesto mutevole, le associazioni nazionali di antropologia, quindi, potrebbero operare una segnalazione al Garante della Privacy sottolineando le criticità incontrate dagli/lle antropologi/ghe al fine di includere nella revisione le nostre necessità.

In questa sezione ho discusso un aspetto specifico (trattamento di categorie speciali di dati) del più ampio processo che mi ha vista impegnata nella formulazione di un protocollo etico, col fine di mettere in evidenza tre aspetti principali, tra loro legati. Il primo è la particolare posizionalità del/lla ricercatore/ice vis a vis i vari enti che lo/la supportano nel processo di valutazione e validazione degli aspetti etici di un progetto europeo. Il/la ricercatore/ice si trova in mezzo a due attori principali: da un lato il proprio ateneo e dall'altra l'ERCEA. Può capitare che gli ostacoli maggiori si trovino nelle negoziazioni con l'ateneo (su cui grava la responsabilità legale) piuttosto che con ERCEA, che nel mio caso, e per il momento, ha mostrato apertura al dialogo e una certa considerazione delle specificità disciplinari. Il tipo di supporto che si riceve dal proprio ateneo può essere quindi variabile e dipende da molti fattori che vanno dalla scala micro dei rapporti interni tra l'ateneo e il/la ricercatore/ice, alle politiche più ampie dell'ateneo in termini di presenza disciplinare e in termini di supporto alla ricerca.

Ciò mi porta al secondo punto, ovvero la disomogeneità delle risposte date, in materia di etica, dai diversi atenei nazionali. Questa disomogeneità dipende dalla diversa organizzazione e competenza, che gli atenei hanno, nella valutazione dei protocolli etici. Per questo motivo, ritengo che sia molto importante attivare un lavoro di informazione e advocacy da parte delle associazioni nazionali di antropologia.

Infine, il terzo punto si riferisce all'interpretabilità del GDPR nella progettazione europea. Il GDPR, prima ancora che essere visto come un insieme di articoli che possono essere messi in discussione solo sul piano legale, va inserito in un sistema più ampio che presenta vari riferimenti, collettivi e dibattiti. In sede di progettazione europea, ERCEA richiede che venga fatta una riflessione critica dei propri procedimenti di ricerca e le risposte sono negoziabili perché è viva la consapevolezza che l'etica è una questione per la quale non si possono dare risposte univoche in termini di 'giusto' o 'sbagliato'.

A questo proposito, nelle sezioni successive illustrerò le mie risposte - ispirate dalla lettura di riferimento già citata più sopra - ad alcune richieste avanzate da ERCEA nella valutazione etica del mio progetto. Queste si riferiscono ad alcuni aspetti che possono essere comuni alla disciplina antropologica. In particolare, discuterò alcuni dei problemi che prestano il fianco a una valutazione attenta delle nostre modalità 
comunicative con i referenti istituzionali ed europei, con particolare riferimento al concetto di 'dati', ma che stimolano anche una riflessione critica delle nostre stesse pratiche di ricerca.

\section{Terze parti} richieste del GDPR, è il fatto che il più delle volte abbiamo a che fare con quelli che vengono definiti 'human participants'. Oltre alle interviste, la nostra metodologia si avvale dell'osservazione partecipante. Sebbene si possano prevedere tutta una serie di precauzioni per tutelare la privacy e la libertà delle persone che si intervistano, $\mathrm{i}$ valutatori normalmente vogliono sapere quali sono le misure previste per la tutela delle 'terze parti', ovvero le persone che rientrano nella nostra osservazione anche se non intervistate direttamente.

Nel caso specifico della mia ricerca, un/a ricercatore/ice può presentare e far firmare il consenso informato agli scienziati intervistati, ma mi veniva richiesto di chiarire come si intendeva tutelare le persone che frequentano occasionalmente il laboratorio o che posso incontrare mentre accompagno gli scienziati alla mensa, a conferenze o incontri di vario tipo. La mia soluzione fu quella di esplicitare che incontri con terze parti sarebbero stati possibili ma che «the focus will be on scientists/professionals and their relation to that third party - not the third party itself». Dopo aver letto il documento promosso dalla Comunità Europea - Research Ethics in Ethnography/Anthropology - mi resi conto che avrei potuto anche aggiungere che l'osservazione di luoghi pubblici dovrebbe essere consentita (al pari della categoria dei giornalisti, per esempio) perché fa parte delle metodologie convenzionali della ricerca etnografica.

Il primo ateneo, però, quello con cui avevo inviato la proposta progettuale, mi disse che dovevo aggiungere anche la frase: «Research participants will be sensitized about the ethical issues related to occasional and unplanned third parties involvement in the project and about the techniques of minimization of risks related to it. These will be illustrated in the information sheet and discussed before any fieldwork will commence». Anche in questo caso, allertare i/le partecipanti alla ricerca sui rischi etici e le tecniche di minimizzazione del rischio nel caso avessimo incontrato qualcuno che non aveva firmato il consenso mi sembrava non solo un formalismo vuoto di sostanza ma anche lesivo della pratica antropologica. Per le ragioni già illustrate più sopra, con rammarico, aggiunsi la frase. Discutendo con Marco su questo aspetto, ed esplorando la possibilità di eliminare tale frase, egli mi rassicurò spiegandomi che il modulo di consenso informato da loro sviluppato include il riferimento all'articolo 6(1,e) del GDPR che regola la liceità del trattamento dei dati personali (cfr. anche Bernes 2020). Il documento, infatti, così recita: «The research activities are conducted by the University in the public interest as part of its official functions, therefore the legal basis for the processing of personal data is represented by art. 6.1.e) of the Regulation ("performance of a task carried out in the public interest")».

\section{Minimizzazione dei dati}

Un altro problema spinoso per la pratica antropologica si riferisce al principio di 'minimizzazione dei dati'. Secondo il GDPR, i dati processati devono essere rilevanti e 
limitati all'obiettivo della ricerca. Questa richiesta è, da una parte, legittima. Faccio un esempio. A volte, mi è capitato di avere la tentazione di inserire in un articolo o in un libro pezzi di un'intervista che, citati come discorso diretto, creavano un forte tono emotivo - quasi scandalistico - capace di mettere in forte risalto conflitti e giudizi. In questi casi faccio sempre uno sforzo per trasformare la frase da discorso diretto a indiretto e, spesso, mi rendo conto che in questa maniera essa perde il suo potenziale dirompente perché è necessario approfondire la contestualizzazione e andare all'origine di tale frase, comprendendone le motivazioni più profonde. In questo modo, si arricchisce l'analisi al di là del livello scandalistico. In questo modo, la richiesta di processare dati rilevanti all'obiettivo della ricerca non solo permette di produrre conoscenza in maniera etica, ma anche in maniera più scientificamente rigorosa.

Dall'altra, però, la richiesta di minimizzazione dei dati può portare a trasfigurazioni della disciplina antropologica. I dati raccolti non possono essere astratti dalla situazione sociale, per sua natura complessa, qualsiasi essa sia. E non è neppure possibile prevedere in anticipo quali dati saranno 'rilevanti', né quale sarà l'obiettivo finale della ricerca. Certo, ogni ricerca è guidata da un obiettivo iniziale, che deve essere ben chiaro, ma questo può (e deve) variare nel corso della ricerca proprio perché il valore del metodo antropologico è quello di adattarsi alle situazioni che si trovano sul campo (che, come normalmente accade, possono essere diverse da quelle previste). Non è quindi possibile definire a priori quali dati saranno raccolti, e quali considerati rilevanti ma ciò sarà un processo che lega il ricercatore al partecipante per tutto l'arco di vita della ricerca (dal suo disegno alla disseminazione dei risultati, e anche oltre).

La richiesta di minimizzazione dei dati, come spesso accade, procede a partire da un'idea di scienza e di ricerca scientifica che prende a riferimento le scienze naturali, il cui procedimento è generalmente quello di verificare delle ipotesi date a priori secondo delle variabili anch'esse definite a priori. È quindi vitale portare avanti un dibattito che renda esplicita ed estenda la legittimità delle nostre pratiche di ricerca. Condivido quindi volentieri (qui sotto) la mia risposta ai valutatori su questo punto, se potrà servire a qualcuno/a. Questa è stata formulata ispirandomi alla letteratura già citata, riassumendola col fine di permettere ai valutatori di apprezzare le specificità del metodo antropologico e armonizzarle con le richieste di minimizzazione dei dati:

Data minimisation principle is valuable both on an ethic and scientific ground. Therefore, I commit myself to process data that will be relevant (in the sense that they will have a rational link to the research purpose, so creating a focused and consistent narrative) and that will be limited to the research's stated aim and objectives. No real names or other unnecessary details will be published or recorded. It is worthy to emphasize, however, that I'll respect these criteria within the conditions set by the nature of ethnographic research. As stated in in the European Association of Social Anthropologists - EASA's Statement on Data Governance in Ethnographic Projects, ethnographic materials, for their very nature, are embedded in particular social contexts. These, by definition, are complex situations. A preliminary (before fieldwork) assessment of how data will be considered relevant and limited applies well to hypothesis-testing research designs but, if stated, risk creating wrong expectations in research participants and problems of fairness in ethnographic research. Ethnography relies not on a design epistemology (verification of hypothesis) but on a discovery epistemology. Its scientific validity depends on the capacity to adapt to unpredictable conditions encountered during research, of course within the limits of law and also of the GDPR legislation. Ethics, in ethnographic research, is not an anticipatory check or an afterthought but is embedded in every stage of the research process, from 
formulating research questions and methodology, negotiating fieldwork and relationships with interlocutors, and managing data, to writing and other forms of representation and professional engagement.

\section{Gestione e protezione dei dati}

Nel GDPR viene dato molto valore ai 'dati'. Ciò non è un caso, ma avviene parallelamente all'incremento del loro valore nell'economia della conoscenza. L'influenza sempre più forte della tecnologia nella scienza (soprattutto quelle naturali) ha trasformato i dati in merce preziosa perché i dati, al giorno d'oggi, producono ricchezza. Viviamo in un'epoca in cui molte delle conoscenze derivano dal processamento dei così chiamati big data, quantità molto alte di dati aggregati secondo algoritmi ad hoc. Sono queste pratiche a costituire il riferimento principale per la regolamentazione sui dati. Nelle scienze 'dure' esiste un doppio registro: chi fa ricerca sa benissimo che anche i dati sono legati a un contesto, dall'altra viene incoraggiato il loro utilizzo in quanto unità autonome, astratte e replicabili. L'incongruenza tra queste due posizioni sta dando luogo a quella che viene definita la 'crisi della riproducibilità' nelle scienze naturali (Baker Penny 2016). In antropologia, invece, non si è mai smesso di riconoscere l'importanza del contesto e dell'interpretazione nella costituzione dei dati. I dati, in antropologia, non possono mai essere 'grezzi' ma sono già e sempre il frutto di un processo di selezione ed interpretazione. Sono, quindi, legati intimamente al/la ricercatore/ice che li ha prodotti. Ciò significa, anche, che non sono alienabili e sono il risultato di un'opera di ingegno che deve essere protetta dal diritto d'autore.

I valutatori dell'ERCEA richiedono informazioni dettagliate su quali siano le misure tecniche e organizzative per salvaguardare i dati e come si garantisca, attraverso queste misure, la libertà e privacy dei/lle partecipanti alla ricerca. Anche in questo caso, trovo che questa richiesta abbia un lato salutare. Essa porta l'attenzione sul valore dei dati, sulla responsabilità che abbiamo in quanto ricercatori/ici di preservare la loro integrità e permettere la loro reperibilità in maniera ordinata. I dati, in fondo, non sono solo 'nostri' ma sono un dono dei/lle partecipanti alla ricerca e in quanto tali dovremmo curarli con le giuste attenzioni. Ma proprio perché i dati in antropologia non sono solo di chi li raccoglie, essi sono in una certa misura co-prodotti. Quello che ho evidenziato nella mia risposta ai valutatori, quindi, è che la co-produzione implica già una considerazione dei diritti dei/lle partecipanti: "Given that anthropological knowledge is co-produced in the ethnographic encounter, that knowledge already includes the safeguarding of participants' rights and freedom." Ovviamente, tale risposta è valida solo se davvero la ricerca si muove attraverso un disegno partecipativo, speso anche sfidando certe abitudini e presupposti che ci vedono autorizzati a non metterci totalmente in gioco nella relazione etnografica (Mosse 2015).

\section{Conclusione}

Gli aspetti etici della ricerca sono un aspetto importante. Non dovrebbero essere considerati come un impiccio e neppure come una battaglia che vede noi, i/le ricercatori/ici opposti/e a amministratori e valutatori. L'etica, se opportunamente approcciata, può costituire uno straordinario stimolo a porci delle domande e, quindi, 
aumentare la qualità delle ricerche che conduciamo, aiutandoci a esplorare le pieghe anche più sottili della nostra relazione con il campo e con i/le partecipanti.

Quello che però ho cercato di evidenziare in questo articolo, è che le risposte che diamo a queste domande non devono essere guidate solo da una dimensione tecnico-legale, né solo dalle richieste dell'apparato amministrativo universitario e istituzionale. $\grave{E}$ importante non cedere - come io ho fatto in un primo momento - alla tentazione di accettare affermazioni che non fanno giustizia alla pratica antropologica e che non mettono neppure in moto un processo di comprensione, da parte degli amministratori e valutatori, della disciplina antropologica. La collaborazione con esperti in materia ci permette di ottemperare ai nostri doveri, ma anche di perfezionare la normativa con osservazioni legate alla prospettiva antropologica. Come ho avuto modo di sottolineare nell'articolo, questa alleanza non è scontata e servono le risorse necessarie per metterla in moto. Serve un'università che dia fondi adeguati al settore amministrativo perché è da lì, nell'università capitalista del 21esimo secolo, che parte la possibilità di avere fondi per la ricerca, e farla bene.

Molti possono convenire sul fatto che l'università neoliberale in cui ci troviamo a lavorare, così improntata ad un'organizzazione aziendale, ha molte pecche. Per un suo rinnovamento, però, ritengo che serva innanzitutto partire dalla lucida analisi di ciò che al momento c'è, cercando degli spazi in cui innestare pratiche e logiche alternative. Il problema, in altri termini, non è tanto l'amministrazione in sé e gli spazi sempre maggiori di influenza che sta guadagnando, ma un sistema accademico trasfigurato. Come rimediare a ciò? La complessità di ogni fenomeno sociale permette di individuare degli spazi di azione in cui ricollegare ciò che è "potenziale» a ciò che è "possibile» (Zigon 2019), persino all'interno del sistema capitalistico neoliberale (Bear et al. 2015). Più che ancorarsi alla sola critica, questa può produrre rinnovamento attraverso una politica sperimentale, fatta di «dispersed, everyday, imperceptible politics» (Papadopoulos 2018: 157) che si innestano nel sistema e i cui effetti sono visibili quando attori sociali convenzionalmente marginali (come lo è l'antropologia rispetto alla 'scienze dure') «emerge in the political scene by changing the very constitution of being» (Papadopoulos 2018: 18). Ciò è possibile se si agisce sia in resistenza ma anche in collaborazione con il sistema, dando così forma a delle relazioni né di mimesi ma neppure di conflitto. La radice del problema potrebbe, quindi, contribuire a innestare i semi di una sua soluzione se, per esempio, la partecipazione a progetti europei sia vissuta come un'occasione per trasformare il sistema dall'interno, in dialogo e con il supporto di associazioni di antropologia nazionali, europee ed internazionali. Come illustrato in questo articolo - che ha sottolineato i momenti di difficoltà e le impasse fare questo da soli è difficile, ed è per questo essenziale costruire delle reti a livello nazionale ma anche internazionale. Nel mio caso, la letteratura a disposizione, per quanto importante, non era abbastanza per provare a rinegoziare le risposte che ho dato. Dopo il supporto dell'ateneo di cui faccio ora parte, e dopo l'incontro SIAC, mi sento più pronta.

L'alternativa, altrimenti, è quella di avallare un discorso che ho sentito spesso circolare: bisogna far buon viso a cattivo gioco, adeguandosi alle richieste del GDPR e dei valutatori - anche se non adeguate - per poi comportarci sul campo 'come sempre abbiamo fatto'. Come antropologi/ghe, però, sappiamo bene che le parole, le risposte che noi diamo nei formulari non sono innocenti ma contengono già una dimensione performativa. Trovo quindi importante non aderire acriticamente a una certa narrativa 
su cosa sia l'etica, cosa siano i dati, cosa siano la libertà e la privacy. Il rifiuto di conformarsi a delle richieste che non tengono conto delle peculiarità della disciplina antropologica non ha il solo scopo di salvaguardare la nostra disciplina e il nostro modo di fare ricerca. Come antropologi/ghe, il nostro intervento nei discorsi sull'etica è fondamentale e ha un valore pubblico che va ben oltre gli interessi personali o disciplinari. L'etica, in fondo, altro non è che una riflessione sulla natura delle relazioni sociali, i rapporti di fiducia, i legami di debito e reciprocità che legano le persone, i non detti. L'antropologia ha quindi un ruolo sociale e pubblico importante nel dibattito sull'etica, probabilmente più di altre discipline.

\section{BIBLIOGRAFIA}

Baker M., Penny D., 2016 «Is there a reproducibility crisis?», in Nature, 533: 452-453.

Bear L. et al., 2015 «Gens: A Feminist Manifesto for the Study of Capitalism», in Theorizing the Contemporary, Fieldsights, March 30. https://staging.culanth.org/fieldsights/gens-a-feministmanifesto-for-the-study-of-capitalism.

Bernes A., 2020 «La protezione dei dati personali nell'attività di ricerca scientifica», in Le Nuove Leggi Civili Commentate, 1: 175-205.

de Koning M. et al., 2019 «Guidelines for anthropological research: Data management, ethics, and integrity», in Ethnography, 20(2): 170-174.

Dilger H., Pels P., Sleeboom-Faulkner M., 2019 «Guidelines for data management and scientific integrity in ethnography», in Ethnography, 20(1): 3-7.

Etzkowitz H., 2013 «Anatomy of the entrepreneurial university», in Social Science Information, 52(3): 486-511.

Mosse D., 2015 «Misunderstood, misrepresented, contested? Anthropological knowledge production in question», in Focaal - Journal of Global and Historical Anthropology, 72: 28-137.

Papadopoulos D., 2018 Experimental Practice: Technoscience, Alterontologies, and More-Than-Social Movements, Duke University Press, Durham.

Pels P. et al., 2018 «Data management in anthropology: the next phase in ethics governance?», in Social Anthropology, 26(3): 391-413.

Slaughter S., Rhoades G., 2004 Academic Capitalism and the New Economy: Markets, State, and Higher Education, Johns Hopkins University Press, Baltimore.

Stoica G. et al., 2019 «Precarity without borders: visions of hope, shared responsibilities and possible responses», in Social Anthropology, 27(S2): 78-96.

Zigon J., 2019 A War on People: Drug User Politics and a New Ethics of Community, University of California Press, Oakland. 


\section{NOTE}

1. https://erc.europa.eu/la-missione/it

2. https://cordis.europa.eu/project/id/949742/it

3. La metagenomica è lo studio genetico di comunità ecologiche di microbi.

4. Il fatto che un documento istituzionale sull'etnografia/antropologia venga affidato a un sociologo meriterebbe una riflessione a parte.

5. Nome anonimizzato

\section{RIASSUNTI}

In questo articolo vengono discussi gli aspetti etici, con particolare riferimento al GDPR, di un studio di caso (finanziamento ERC) di progettazione europea. Attraverso una ricostruzione etnografica del processo, delle negoziazioni, dei dubbi e delle frustrazioni, il tentativo è quello di decostruire l'etica come un campo che pertiene strettamente alla dimensione legale o amministrativa, ma mostrare come tali dimensioni dipendano da elementi socio-politici locali, nazionali, europei e globali. Nell'articolo viene data rilevanza a due aspetti principali. Uno è il significato da dare all'etica, come insieme di norme rigide oppure come un incoraggiamento ad aprire un discorso sull'etica, significato da cui deriva il posizionamento della comunità antropologica. L'altro aspetto è quello delle peculiarità della ricerca antropologica rispetto alla definizione normativa di 'dati'.

This article discusses the ethical aspects, with particular reference to the GDPR, of a case study (ERC funding) of European project. Through an ethnographic reconstruction of the process, negotiations, doubts and frustrations, the attempt of this article is to deconstruct ethics as a field that pertains strictly to the legal or administrative dimension, showing how these dimensions are intertwined with socio-political elements at the local, national, European and global level. The article emphasizes two main aspects. One is the meaning to be given to ethics, as a set of rigid rules or as an encouragement to open a discourse on ethics, from which derives the positioning of the anthropological community. The other aspect discusses the peculiarities of anthropological research with respect to the normative definition of 'data'.

\section{INDICE}

Keywords : GDPR, ERC, data, informed consent, special categories of data Parole chiave : GDPR, ERC, etica, dati, categorie speciali di dati

\section{AUTORE}

\section{ROBERTA RAFFAETÀ}

Dipartimento di Filosofia e Beni Culturali, Università Ca' Foscari Venezia roberta.raffaeta@unive.it 